\title{
Performance evaluation of the croissant production line with reparable machines
}

\author{
Panagiotis H. Tsarouhas
}

Received: 19 December 2013/Accepted: 22 August 2014/Published online: 13 September 2014

(C) The Author(s) 2014. This article is published with open access at Springerlink.com

\begin{abstract}
In this study, the analytical probability models for an automated serial production system, bufferless that consists of $n$-machines in series with common transfer mechanism and control system was developed. Both time to failure and time to repair a failure are assumed to follow exponential distribution. Applying those models, the effect of system parameters on system performance in actual croissant production line was studied. The production line consists of six workstations with different numbers of reparable machines in series. Mathematical models of the croissant production line have been developed using Markov process. The strength of this study is in the classification of the whole system in states, representing failures of different machines. Failure and repair data from the actual production environment have been used to estimate reliability and maintainability for each machine, workstation, and the entire line is based on analytical models. The analysis provides a useful insight into the system's behaviour, helps to find design inherent faults and suggests optimal modifications to upgrade the system and improve its performance.
\end{abstract}

Keywords Manufacturing systems - Performance evaluation - Markov chain - Croissant production line . Reparable machines

P. H. Tsarouhas $(\bowtie)$

Department of Supply Chain Management and Logistics,

Technological Educational Institute of Central Macedonia,

Kanellopoulou 2, 60100 Katerini, Greece

e-mail: ptsarouhas@teicm.gr

\section{Introduction}

An automated production system is a set of machines, transportation elements, buffers, automations and a control system used simultaneously to produce a desired product. The main target of the production system is to operate appropriately with the maximal production rate or capacity and acceptable quality of the products. The major problem that impedes the performance of production systems is the lack of reliability of the machines. There are many studies on performance evaluation of production systems subjected to failures (Buzacott and Shanthikumar 1993; Dallery and Gershwin 1992; Xie 1993; Govil and Fu 1999). An unscheduled failure of a machine may affect the performance of the rest of the machines, both upstream and downstream, thereby blocking the former and starving the latter (Chiang et al. 1998). The importance in promoting, sustaining industries, manufacturing systems and economy through reliability measurement has become an area of interest (Yusuf 2014).

The contributions of this paper are twofold. The first is to develop analytical probability models for an automated, bufferless serial production system that consists of $n$ machines in series with common transfer mechanism and control system. The steady-state probability models of operation and failure for any individual machine, workstation and for the entire production system, using the Markov approach, were evaluated. The second is to perform a numerical investigation of the effect of the system parameters on system performance in the actual croissant production line. Moreover, the analysis can help to increase the efficiency and the quality of the production system.

The rest of this manuscript is organized as follows: (a) in the "Literature review" is presented; (b) in "Methods", "Reliability, availability and maintainability equations", 
and "Mathematical formulation of the system" are described. (c) In the "Results and discussion" are presented with a numerical example, and finally, (d) in the "Conclusions" are drawn.

\section{Literature review}

The literature survey on production systems is quite vast; Karim et al. (2008) presented the results of a study conducted to identify some of the effective manufacturing practices that have a significant impact on manufacturing performance. Patti and Watson (2010) reported that combinations of different downtime such as mean time between failures (frequency) and mean time to repair (duration) affect serial production lines differently even when the total downtime remains equal. Freiheit et al. (2004) developed models to predict the productivity of pure serial and parallel-serial bufferless production lines with reserve capacity that is the provision of flexible stand by machines capable of performing any operation carried out in the main production line. Faghihinia and Mollaverdi (2012) presented a multi-criterion decision-aided maintenance model with three criteria that have more influence on decision making: reliability, maintenance cost and maintenance downtime. Adamyan and He (2002) presented a methodology to identify the sequences of failures and probability of their occurrence in an automated manufacturing system. Glassey and Hong (1993) developed an efficient method to analyse the behaviour of an unreliable $n$-stage transfer line with $(n-1)$ finite inter-stage storage buffers. The method is based on the thorough examination of the steady-state behaviour of the $n$-stage line and the decomposed lines and the relationship between the failure and repair rates of the individual stages and the aggregate stages. This method was improved later on by Burman et al. (1998).

There are several studies related to reliability, availability and maintainability of manufacturing systems. Cockerill (1990) has presented a Reliability, Availability and Maintainability-RAM analysis of a turbine-generator system. Tsarouhas (2012) reviewed the reliability, availability and maintainability (RAM) analysis in the food industries and aims to identify the critical points of the production systems that should be improved by the operational performance and the maintenance effectiveness. Collas (1994) has given a simple methodology for assessing the complex system availability and reliability. Koulamas (1992) and Savsar (2000) developed Markov models to study the effects of tool failures on system performance measures for a flexible manufacturing cell with a single machine served by a robot for part loading/unloading and a pallet for part transfers to estimate the reliability and maintenance. Hassett et al. (1995) have combined time varying failure rates and Markov chain analysis to obtain hybrid reliability and availability analysis. Rezg et al. (2004) proposed an integrated method for preventive maintenance and inventory control of a production line, composed of $n$ machines subject to failures without intermediate buffers. Marseguerra et al. (2004) calculated optimal reliability and availability of uncertain systems via multi-objective genetic algorithms. Born and Criscimagna (1995) developed a methodology to evaluate the need of reliability, maintainability and diagnostics for translation processes. Sharma et al. (2008) proposed a methodological and structured framework that makes use of both qualitative and quantitative techniques aiming at risk and reliability analysis of the system. Tsarouhas (2013) studied the reliability and maintainability analysis, which are the fundamental issues for the operation management of a dialysis system. Markeset and Kumar (2001) have discussed the application of reliability, maintainability and risk analysis methods to minimize life cycle cost of the system. Tatry et al. (1997) have presented an advance study on reliability, availability, maintainability and safety-RAMS for a reusable launch vehicle. Lin (2009) studied system reliability evaluation for a multistate supply chain network with failure nodes using minimal paths.

Furthermore, stochastic models are displayed under a stochastic environment with tool failure and replacement consideration. Simeu-Abazi et al. (1997) applied decomposition and iterative analysis of Markov chains to obtain numerical solutions for the reliability and dependability of manufacturing systems. Lai et al. (2002) have developed Markov model and equations for distributed software/ hardware systems to obtain the steady-state availability. Kim and Geshwin (2005) proposed a new model for machines with both quality and operational failures and developed a Markov process-based method for performance analysis of production systems consisting of such machine. Taghizadeh and Hafezi (2012) used supply chain operational reference for investigating the reliability evaluation of available relationships in supply chain. Tan (1999) proposed a method to calculate both average and variance of transient throughput by further assuming that the series system at steady-state is in an irreducible Markov chain. Golrizgashti (2014) proposed a developed balanced scorecard approach to measure supply chain performance with the aim of creating more value in manufacturing and business operations. Hou and Okogbaa (2002) proposed a simplified availability modelling worksheet (SAMOW), a computational tool that incorporates Markov analysis and reliability block diagram methodologies to model and analyse the availability of a typical end-to-end solution consisting of multiple complex component systems, where the failure of each component system is attributed to software failures and hardware failures. Srinivasa Rao and Naikan (2014) proposed a hybrid approach called as 
Markov system dynamics (MSD) approach which combines the Markov approach with system dynamics simulation approach for reliability analysis and to study the dynamic behaviour of systems.

In many high-speed lines, especially in the process industries, it may not be possible to store in-process material, because buffers may hurt the quality of the material by allowing it to deteriorate over time (Liberopoulos et al. 2007). For these reasons, high-speed lines generally do not have buffers between workstations. Dogan and Altiok (1998) mentioned that in a pharmaceutical transfer line with several workstations in series connected with conveyor segments and buffers between workstations, when a workstation fails, the part in it is scrapped. Chen and Yuan (2004) presented a method to calculate and estimate the performance indices such as the mean and variance of the transient throughput and the probability that the total outputs will meet the demand on time for a series of unreliable machines with the same production rate and without intermediate buffers. Raissi and Gatmiry (2012) addressed a systematic method for measuring multi-stage service reliability function using failure rate analysis beside a systematic Six Sigma approach to improve total system reliability. Jin et al. (2011) proposed a Six Sigma based framework to deploy high product reliability commitment in distributed subcontractor manufacturing processes. Dhouib et al. (2008) studied the steady-state availability and throughput of production lines without buffers composed of several serial machines subject to random operation-dependent failures.

\section{Methods}

Reliability, availability and maintainability equations

Reliability of a system is the probability that the item will perform its intended function throughout a specified time period when operated in a normal environment (Brischke and Murthy 2003; Dhillon 2006; Pecht 2009). The reliability of a system at operational time $t$ can be expressed as

$R(t)=P(T \geq t)=\exp (-\lambda t)$,

where the continuous random variable $T$ is the time to failure and $\lambda$ is the constant failure rate of the system. The parameters of reliability are mean time to failure/repair, failure/repair rate and maximum number of failures in a specific time interval:

Reliability + Unreliability $=1$

To describe the failure and repair process of the production systems we consider a failure distribution that has a constant failure and repair rate representing the exponential probability distribution. Some of the reasons for failure occurrence may be undetectable defects, abuse, low-safety factor, etc.

The mean time to failure (MTTF) is defined by

MTTF $=\int_{0}^{\infty} R(t) \mathrm{d} t=\frac{1}{\lambda}$.

The mean time to repair is defined by

MTTR $=\int_{0}^{\infty}(1-H(t)) \mathrm{d} t=\frac{1}{r}$,

where $H(t)$ is the cumulative distribution function of the time to repair a failed system, and $r$ is the constant repair rate of the system. Eqations (2) and (3) are valid for a single component.

The maintainability quantifies the repair time for the failed system and is defined as the probability that the failed system will be restored to its satisfactory operational state when maintenance is performed. Maintainability is related to the duration of outages. The most important measure of maintainability is the mean time to repair (MTTR) that focuses on downtime. In the case where the repair time is exponentially distributed,

$M(t)=1-\exp (-t /$ MTTR $)$.

Also Maintainability + Unmaintainability $=1$ is valid.

Availability is the probability that a system is available for use when required. The availability depends on both reliability and maintainability because first of all failure and repair distribution must be defined. The average availability over the interval $[0, \mathrm{~T}]$ is defined as follows (Ebeling 1997):

$A(T)=(1 / T) \int_{0}^{T} A(t) \mathrm{d} t$.

The steady-state or long-run equilibrium availability is defined

$A=\lim _{T \rightarrow \infty} A(T)=\frac{\mathrm{MTTF}}{\mathrm{MTTR}+\mathrm{MTTF}}$

and Availability + Unavailability $=1$

High availability means high reliability with suitable maintainability which characterises the efficiency of the entire system. Therefore, system availability can never be less than system reliability.

Mathematical formulation of the system

It is considered that a production line where the probability of a transition will undergo from one state to another state 


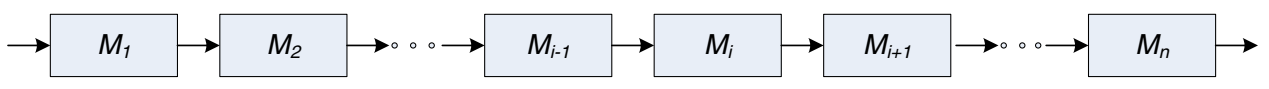

Fig. 1 Schematic representation of a bufferless production line with $n$-machines in series

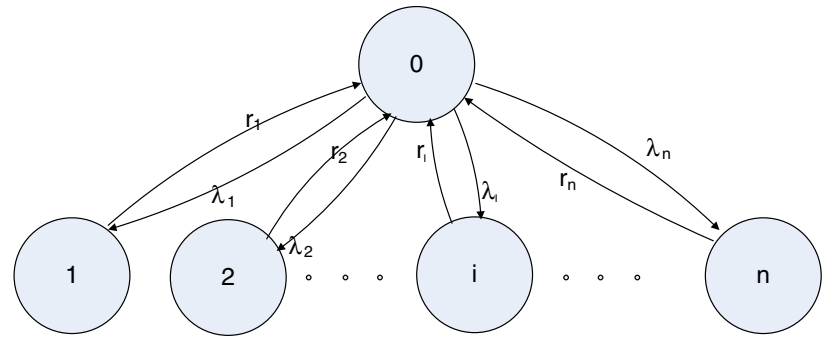

Fig. 2 Transition diagram for $n$-machines

depends only on the current state of the line (Markov process assumption). Therefore, the exponential distribution satisfies the times of failure and repair of the machines. The line which is considered as a system consists of $n$-machines in series with no buffers between them, see Fig. 1. It was also assumed the process is stationary, meaning that the transition probabilities do not change with time. In this study we model a machine as a discrete state, continuous time Markov process. The state transitions of the machine denote the operational state of the machine with 'up' and the under repair state of the machine with 'down'.

In addition, the following are considered: state 0: all machines are up; state 1: only the machine 1 is down, whereas the rest of the machines are up; state 2: only the machine 2 is down, whereas the rest of the machines are up, etc. In the case of a single machine, when a machine is in state 0 , it can fail (i.e. mechanical, electrical, pneumatic causes or human error) and goes to state 1 with probability or failure rate $\lambda$, which is the reciprocal of the Mean Time to Failure (MTTF). After that the maintenance staff fixes it, and the machine goes back to operational state 0 with probability or repair rate $r$, which is the reciprocal of the Mean Time to Repair (MTTR).

The production system in study consists of $n$-machines in series, a failure of $M_{1}$ or $M_{2} \cdots$ or $M_{n}$ obliges the system (or workstation) to stop. The system is operated when all the machines are 'up', state 0 ; otherwise, a failure in any machine (i.e. $M_{i}$ ) can make the system 'down', state $i$ where $i=1,2,3, \ldots n$ (see Fig. 2).

The transition diagram of the system is shown in Fig. 2, and for state 0 the following can be derived:

$$
\begin{aligned}
& P_{0}(t+\Delta t)=P_{0}(t)-\left[\lambda_{1} \Delta t P_{0}(t)+\lambda_{2} \Delta t P_{0}(t)+\cdots\right. \\
& \left.\quad+\lambda_{n} \Delta t P_{0}(t)\right] \\
& \quad+\left[r_{1} \Delta t P_{1}(t)+r_{2} \Delta t P_{2}(t)+\cdots+r_{n} \Delta t P_{n}(t)\right]
\end{aligned}
$$

which states that the probability of the system being in state 0 at time $t+\Delta t$ equals to the probability of it being in state 0 at time $t$ minus the probability of it is being in state 0 at time $t$ and the probability of transitioning $\left(\lambda_{i}\right.$ $\Delta t$ ) to whichever state $1,2, \ldots$ or $n$ in time $\Delta t$, plus the probability of its being in state $1,2, \ldots$ or $n$ at time $t$ and making a transition $\left(r_{i} \Delta t\right)$ to state 0 in time $\Delta t$. Similarly, for state 1 ,

$P_{1}(t+\Delta t)=P_{1}(t)+\lambda_{1} \Delta t P_{0}(t)-r_{1} \Delta t P_{1}(t)$

for state 2 ,

$P_{2}(t+\Delta t)=P_{2}(t)+\lambda_{2} \Delta t P_{0}(t)-r_{2} \Delta t P_{2}(t) ;$

for state 3 ,

$P_{3}(t+\Delta t)=P_{3}(t)+\lambda_{3} \Delta t P_{0}(t)-r_{3} \Delta t P_{2}(t)$

... etc., and for state $n$,

$P_{n}(t+\Delta t)=P_{n}(t)+\lambda_{n} \Delta t P_{0}(t)-r_{n} \Delta t P_{n}(t)$

The Eq. (7) can be rewritten as

$$
\begin{aligned}
\frac{P_{0}(t+\Delta t)-P_{0}(t)}{\Delta t}= & -\left[\lambda_{1}+\lambda_{2}+\cdots+\lambda_{n}\right] P_{0}(t) \\
& +r_{1} P_{1}(t)+r_{2} P_{2}(t)+\cdots+r_{n} P_{n}(t)
\end{aligned}
$$

In the limiting case it becomes

$$
\begin{aligned}
& \lim _{\Delta t \rightarrow \infty} \frac{P_{0}(t+\Delta t)-P_{0}(t)}{\Delta t} \\
& \quad=\frac{\mathrm{d} P_{0}(t)}{\mathrm{d} t}=-\left[\lambda_{1}+\lambda_{2}+\cdots+\lambda_{n}\right] P_{0}(t) \\
& \quad+r_{1} P_{1}(t)+r_{2} P_{2}(t)+\cdots+r_{n} \Delta P_{n}(t)
\end{aligned}
$$

Similarly, for Eqs. (8), (9), (10) and (11),

$\frac{\mathrm{d} P_{1}(t)}{\mathrm{d} t}=\lambda_{1} P_{0}(t)-r_{1} P_{1}(t)$

$\frac{\mathrm{d} P_{2}(t)}{\mathrm{d} t}=\lambda_{2} P_{0}(t)-r_{2} P_{2}(t)$

$\frac{\mathrm{d} P_{3}(t)}{\mathrm{d} t}=\lambda_{3} P_{0}(t)-r_{3} P_{3}(t)$

$\frac{\mathrm{d} P_{n}(t)}{\mathrm{d} t}=\lambda_{n} P_{0}(t)-r_{n} P_{n}(t)$

The transition rates can be expressed in form of a transition matrix as follows: 


$$
\left[\begin{array}{c}
\mathrm{d} / \mathrm{d} t P_{0}(t) \\
\mathrm{d} / \mathrm{d} t P_{1}(t) \\
\mathrm{d} / \mathrm{d} t P_{2}(t) \\
\mathrm{d} / \mathrm{d} t P_{3}(t) \\
\cdot \\
\cdot \\
\mathrm{d} / \mathrm{d} t P_{n}(t)
\end{array}\right]=\left(\begin{array}{ccccccc}
-\left(\lambda_{1}+\lambda_{2}+\cdots+\lambda_{n}\right) & r_{1} & r_{2} & r_{3} & \cdot & \cdot & r_{n} \\
\lambda_{1} & -r_{1} & 0 & 0 & \cdot & \cdot & 0 \\
\lambda_{2} & 0 & -r_{2} & 0 & \cdot & \cdot & 0 \\
\lambda_{3} & 0 & 0 & -r_{3} & \cdot & \cdot & \cdot \\
\cdot & \cdot & \cdot & \cdot & \cdot & \cdot & \cdot \\
\cdot & \cdot & \cdot & \cdot & \cdot & \cdot & \cdot \\
\lambda_{n} & 0 & 0 & 0 & \cdot & \cdot & -r_{n}
\end{array}\right)\left[\begin{array}{c}
P_{0}(t) \\
P_{1}(t) \\
P_{2}(t) \\
P_{3}(t) \\
\cdot \\
\cdot \\
P_{n}(t)
\end{array}\right]
$$

The system must be one of the $n$ states at any given time. Consequently,

$P_{0}(t)+P_{1}(t)+\cdots+P_{n}(t)=1$

By solving Eqs. (13)-(18) for steady state as $t \rightarrow \infty$ and $\mathrm{d} P(t) / \mathrm{d} t \rightarrow 0$, we obtain the state probabilities

$P_{0}=\left(1+\sum_{i=1}^{n} \frac{\lambda_{i}}{r_{i}}\right)^{-1}$

$P_{1}=\left(1+\frac{r_{1}}{\lambda_{1}}\left(1+\frac{\lambda_{2}}{r_{2}}+\cdots+\frac{\lambda_{n}}{r_{n}}\right)\right)^{-1}$

$P_{2}=\left(1+\frac{r_{2}}{\lambda_{2}}\left(1+\frac{\lambda_{1}}{r_{1}}+\frac{\lambda_{3}}{r_{3}}+\cdots+\frac{\lambda_{n}}{r_{n}}\right)\right)^{-1}$

$P_{3}=\left(1+\frac{r_{3}}{\lambda_{3}}\left(1+\frac{\lambda_{1}}{r_{1}}+\frac{\lambda_{2}}{r_{2}}+\frac{\lambda_{4}}{r_{4}}+\cdots+\frac{\lambda_{n}}{r_{n}}\right)\right)^{-1}$

$P_{n}=\left(1+\frac{r_{n}}{\lambda_{n}}\left(1+\frac{\lambda_{1}}{r_{1}}+\frac{\lambda_{2}}{r_{2}}+\frac{\lambda_{3}}{r_{3}}+\cdots+\frac{\lambda_{n-1}}{r_{n-1}}\right)\right)^{-1}$

In general form the Eqs. 20-23 can be expressed as

$P_{i}=\left[1+\frac{r_{i}}{\lambda_{i}}\left(1+\sum_{\substack{j \neq i \\ 1 \leq j \leq n}} \frac{\lambda_{j}}{r_{j}}\right)\right]^{-1}$, for $i=1,2, \ldots n$

Equation (24) represents analytically the failure probability for individual machine with respect to the failure and repair rate of the rest of the machines' system.

Thus, the availability and unavailability of the system, respectively, are

$$
\begin{aligned}
A_{s}= & P_{0}=\left(1+\sum_{i=1}^{n} \frac{\lambda_{i}}{r_{i}}\right)^{-1} \\
\mathrm{UA}_{s} & =P_{1}+P_{2}+P_{3}+\cdots+P_{n}=\sum_{i=1}^{n} P_{i} \\
& =\sum_{i=1}^{n}\left[1+\frac{r_{i}}{\lambda_{i}}\left(1+\sum_{\substack{j \neq i \\
1 \leq j \leq n}} \frac{\lambda_{j}}{r_{j}}\right)\right]^{-1}
\end{aligned}
$$

Equation (25) has been also proposed, in homogeneous cases by researchers (Buzacott 1968; Buzacott and Shanthikumar 1993; Gershwin 1994; and Papadopoulos and Heavey 1996), whereas Eq. (26) is the unavailability that is the sum of the failure probabilities for each machine of the system.

\section{Results and discussion}

The advantage of the proposed method is to estimate the production system parameters (i.e. reliability availability and maintainability) on system performance in the actual croissant production line. The analysis provides a useful insight into the system's behaviour and helps to find design inherent faults and to suggest optimal modifications to upgrade the system and improve its performance. Then, it re-estimates the system parameters of the production line to measure its efficiency and productivity within the frame of total quality management (TQM) principles.

Numerical example

\section{Description of croissant production system}

The croissant line consists of six independent workstations in series integrated into one system with a common transfer mechanism and control system. Each workstation consists of one-three machines connected in series. The movement of material between stations is performed automatically with mechanical means. There are six distinct stages in croissant production: kneading, forming, proofing, baking, coolingfilling and wrapping. The process flow of the line is as follows (see Fig. 3).

In workstation 1 (kneading), flour from silo and water are automatically fed into the removable bowl of the spiral kneading machine. After the dough is kneaded, the bowl is unloaded from the spiral machine and loaded onto the elevator-tipping device that lifts it and tips it to moisten the dough into the dough extruder of the lamination machine in the next workstation. 


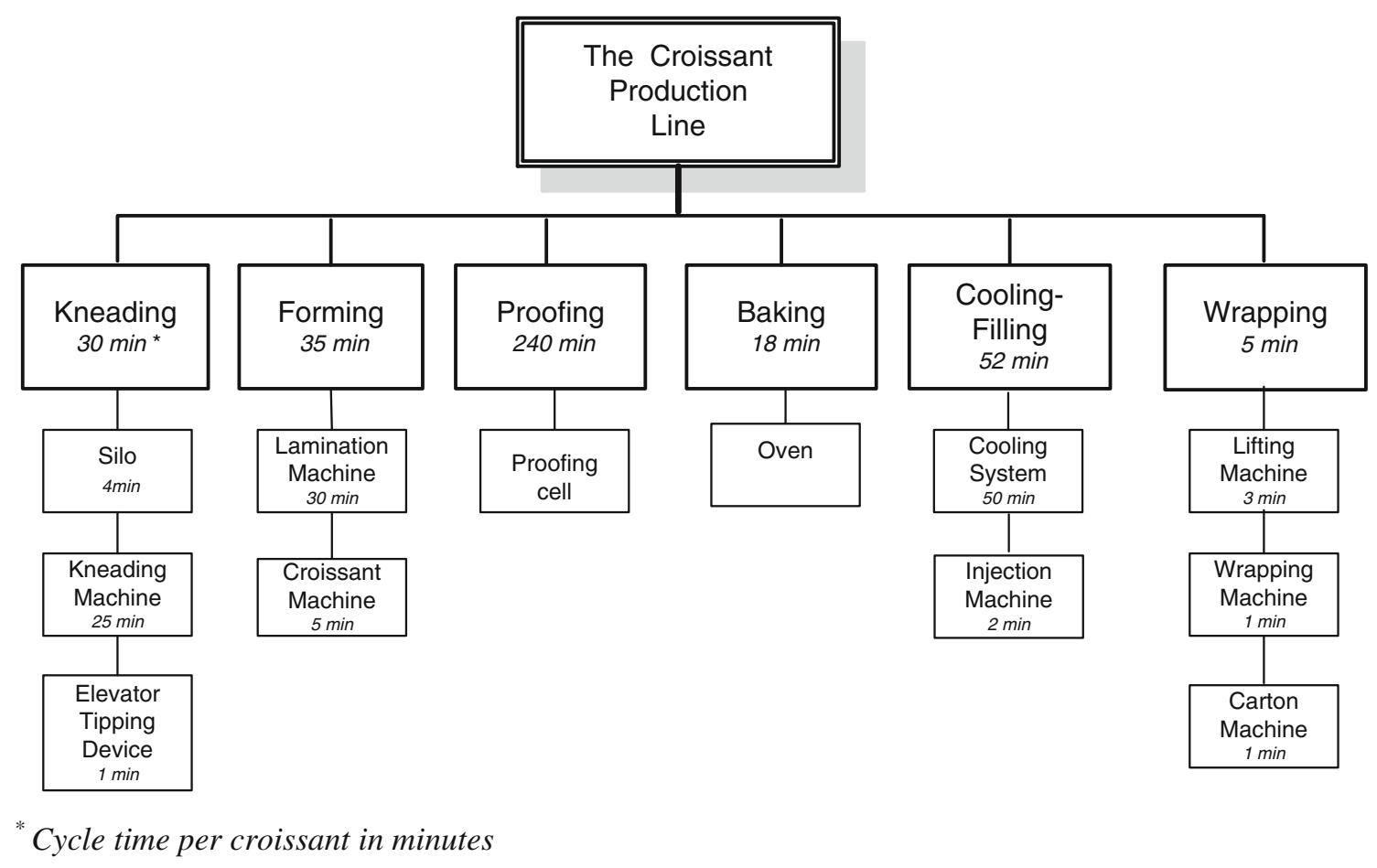

Fig. 3 A schematic presentation of croissant production line

In workstation 2 (forming), the dough fed into the lamination machine is laminated, folded, reduced in thickness by a multiroller and refolded a few times by a retracting unit to form a multilayered sheet. The multilayered dough is fed into the croissant-making machine, which cuts it into pieces of triangular shape. Finally, the triangles are rolled up and formed into crescents. At the exit of the croissant-making machine, the croissants are laid onto metal baking pans, and the pans are inserted into carts.

In workstation 3 (proofing), the carts are automatically pushed into the proofing cell, where they remain under strict uniform temperature and humidity conditions for 240 min so that croissants can rise to their final size.

In workstation 4 (baking), the carts are automatically pushed from the proofing cell to the oven. The baking pans are unloaded from the carts and are placed onto a metal conveyor that passes through the oven. The baking pans remain in the oven for $18 \mathrm{~min}$ until the croissants are fully baked.

In workstation 5 (cooling-filling), the baking pans with the baked croissant remain on the conveyor and follow a trajectory (cooling system) for $50 \mathrm{~min}$ to cool the croissants down and avoid any condensation phenomena (drops) after packaging. The croissants may then be filled with an injecting machine.

In workstation 6 (wrapping), the croissants are automatically lifted from the trays and are flow-packed and sealed with an electronic wrapping machine. The final products are picked up and placed in cartons. The filled cartons are placed on a different conveyor that takes them to a worker who stacks them onto palettes and transfers them to the finished-goods warehouse. The empty baking pans are automatically returned to the croissant-making machine.

Calculation of reliability, availability and maintainability for croissant line

The croissant production line operates for 8-h shifts during each workday and usually pauses during the weekends. From the technical department we collected data that covered a period of 16 months. The data are records of failures that the maintenance staff kept during each shift. During this period the croissant production line operated for $24 \mathrm{~h}$ per day in three 8-h shifts during each day, for a total of 320 working days. The records included the failures that had occurred during each shift, the action taken to repair the failure, the down time and the exact time of failure. Table 1 shows the failure rate $\lambda_{i, j}$ and repair rate $r_{i, j}$, for machine $i$ at workstation $j$.

\section{Workstation 1}

The $\mathrm{WS}_{1}$ consists of 3 machines in series; a failure of $M_{1.1}$ or $M_{2.1}$ or $M_{3.1}$ constrains the workstation to stop. Using 
Table 1 Failure and repair rate for all the machines of croissant production line

\begin{tabular}{llll}
\hline Workstation & Machine & $\lambda_{i, j}($ failures $/ \mathrm{h})$ & $r_{i, j}$ (repairs/h) \\
\hline WS1 & M.1.1 & 0.00890 & 4.6512 \\
& M.2.1 & 0.04100 & 0.7491 \\
WS2 & M.3.1 & 0.00010 & 0.9901 \\
& M.1.2 & 0.09800 & 2.9762 \\
WS3 & M.2.2 & 0.00739 & 0.7994 \\
WS4 & M.1.3 & 0.00946 & 1.1751 \\
WS5 & M.1.4 & 0.00299 & 0.3044 \\
& M.1.5 & 0.02006 & 0.6988 \\
WS6 & M.2.5 & 0.00957 & 1.9531 \\
& M.1.6 & 0.00694 & 2.1882 \\
& M.2.6 & 0.00196 & 0.6523 \\
& M.3.6 & 0.01690 & 1.6000 \\
\hline
\end{tabular}

the Eq. (19) for state 0, and the Eq. (24) for state 1, 2 and 3 as $t \rightarrow \infty$, the following equations can be derived:

$$
\begin{aligned}
& P_{0, \mathrm{WS}_{1}}=\left(1+\sum_{i=1}^{3} \frac{\lambda_{i, 1}}{r_{i, 1}}\right)^{-1} \\
& P_{1, \mathrm{WS}_{1}}=\left(1+\frac{r_{1,1}}{\lambda_{1,1}}\left(1+\frac{\lambda_{2,1}}{r_{2,1}}+\frac{\lambda_{3,1}}{r_{3,1}}\right)\right)^{-1} \\
& P_{2, \mathrm{WS}_{1}}=\left(1+\frac{r_{2,1}}{\lambda_{2,1}}\left(1+\frac{\lambda_{1,1}}{r_{1,1}}+\frac{\lambda_{3,1}}{r_{3,1}}\right)\right)^{-1} \\
& P_{3, \mathrm{WS}_{1}}=\left(1+\frac{r_{3,1}}{\lambda_{3,1}}\left(1+\frac{\lambda_{1,1}}{r_{1,1}}+\frac{\lambda_{2,1}}{r_{2,1}}\right)\right)^{-1} .
\end{aligned}
$$

From Table 1, substituting the values of $\lambda_{i, 1}$ and $r_{i, 1}$ we found the following:

$$
P_{0, \mathrm{ws} 1}=0.946298058, \quad P_{1, \mathrm{ws} 1}=0.001810741,
$$

$P_{2, \mathrm{wS} 1}=0.051795624, P_{3, \mathrm{ws} 1}=9.55761 \mathrm{E}-05$. Therefore, from Eqs. (25) and (26), the availability and the unavailability of the $\mathrm{WS}_{1}$ are $A_{\mathrm{WS} 1}=94.63 \%$ and $\mathrm{UA}_{\mathrm{WS} 1}=5.37 \%$, respectively.

From Eq. (5) it is easy to deduce that

$\mathrm{MTTR}_{\mathrm{WS}_{1}}=\frac{1-A_{\mathrm{WS}_{1}}}{A_{\mathrm{WS}_{1}}} \mathrm{MTTF}_{\mathrm{WS}_{1}}$,

where $\quad \mathrm{MTTF}_{\mathrm{WS}_{1}}=\frac{1}{\Gamma^{3} \lambda^{21}}=\frac{1}{0.05}=20 h ; \quad$ then $\mathrm{MTTR}_{\mathrm{WS} 1}=1.135 \mathrm{~h}$ and $\sum_{\mathrm{WS} 1} \stackrel{\lambda_{i, 1}}{=} 0.881$

The reliability and the maintainability of the $\mathrm{WS}_{1}$ are $R_{\mathrm{WS}_{1}}(t)=\exp [-0.05 t]$ and $M_{\mathrm{WS}_{1}}(t)=1-\exp [-0.881 t]$, respectively.

\section{Workstation 2}

$\mathrm{WS}_{2}$ consists of 2 machines in series; a failure of $M_{1.2}$ or $M_{2.2}$ constrains the workstation to stop. Using the Eqs. (19) and (24) for state 0,1 and 2 as $t \rightarrow \infty$, the following equations can be derived:

$$
\begin{aligned}
& P_{0, \mathrm{WS}_{2}}=\left(1+\sum_{i=1}^{2} \frac{\lambda_{i, 2}}{r_{i, 2}}\right)^{-1} \\
& P_{1, \mathrm{WS}_{2}}=\left(1+\frac{r_{1,2}}{\lambda_{1,2}}\left(1+\frac{\lambda_{2,2}}{r_{2,2}}\right)\right)^{-1} \\
& P_{2, \mathrm{WS}_{2}}=\left(1+\frac{r_{2,2}}{\lambda_{2,2}}\left(1+\frac{\lambda_{1,2}}{r_{1,2}}\right)\right)^{-1}
\end{aligned}
$$

From Table 1, substituting the values of $\lambda_{i, 2}$ and $r_{i, 2}$ we found: $\quad P_{0, \mathrm{wS} 2}=0.95953369, \quad P_{1, \mathrm{wS} 2}=0.03159553$, $P_{2, \text { ws2 }}=0.00887078$. Therefore, from Eqs. (25) and (26), the availability and the unavailability of the $\mathrm{WS}_{2}$ are $A_{\mathrm{WS} 2}=95.95 \%$ and $\mathrm{UA}_{\mathrm{WS} 2}=4.05 \%$, respectively.

The reliability and the maintainability of the $\mathrm{WS}_{2}$ are: $R_{\mathrm{WS}_{2}}(t)=\exp [-0.105 t]$ and $M_{\mathrm{WS}_{2}}(t)=1-\exp [-2.49 t]$, respectively, where MTTF $_{\mathrm{WS}_{2}}=\frac{1}{\sum_{i=1}^{2} \lambda_{i, 2}}=\frac{1}{0.105}=9.49 \mathrm{~h}$ and $\mathrm{MTTR}_{\mathrm{WS} 2}=0.40 \mathrm{~h}$ and $r_{\mathrm{WS} 2}=2.49$.

\section{Workstation 3}

$\mathrm{WS}_{3}$ consists of 1 machine'; a failure of $M_{1.3}$ constrains the workstation to stop. Using Eqs. (19) and (24) for state 0 and 1 , respectively, as $t \rightarrow \infty$, the following equations can be derived:

$P_{0, \mathrm{wS}_{3}}=\left(1+\frac{\lambda_{1,3}}{r_{1,3}}\right)^{-1}$

$P_{1, \mathrm{WS}_{3}}=\left(1+\frac{r_{1,3}}{\lambda_{1,3}}\right)^{-1}$.

From Table 1, substituting the values of $\lambda_{3,1}$ and $r_{3,1}$ we found the following:

$P_{0, \mathrm{wS} 3}=0.992014, \quad P_{1, \mathrm{ws} 3}=0.007986$. Therefore, from Eqs. (25) and (26), the availability and the unavailability of the $\mathrm{WS}_{3}$ are $A_{\mathrm{WS} 3}=99.20 \%$ and $\mathrm{UA}_{\mathrm{WS} 3}=0.8 \%$, respectively.

The reliability and the maintainability of the $\mathrm{WS}_{3}$ are: $R_{\mathrm{WS}_{3}}(t)=\exp [-0.00946 t]$ and $M_{\mathrm{WS}_{3}}(t)=1-\exp [-1.17 t]$, respectively, where $\mathrm{MTTF}_{\mathrm{WS}_{3}}=\frac{1}{\lambda}=\frac{1}{0.00946}=105.70 \mathrm{~h}$ and $\mathrm{MTTR}_{\mathrm{WS} 3}=0.852 \mathrm{~h}$ and $r_{\mathrm{WS} 3}=1.17$.

\section{Workstation 4}

$\mathrm{WS}_{4}$ consists of 1 machine as $\mathrm{WS}_{3}$; a failure of $M_{1.4}$ constrains the workstation to stop.

From Table 1, substituting the values of $\lambda_{1,4}$ and $r_{1,4}$ in Eqs. (19) and (24), we found that

$$
P_{0, \mathrm{wS} 4}=0.990273, P_{1, \mathrm{wS} 4}=0.009727 \text {. }
$$


Therefore, from Eqs. (25) and (26), the availability and the unavailability of the $\mathrm{WS}_{4}$ are $A_{\mathrm{WS} 4}=99.02 \%$ and $\mathrm{UA}_{\mathrm{WS} 4}=0.98 \%$, respectively.

The reliability and the maintainability of the $\mathrm{WS}_{4}$ are: $R_{\mathrm{WS}_{4}}(t)=\exp [-0.00299 t]$ and $M_{\mathrm{WS}_{4}}(t)=1-\exp [-0.302 t]$, respectively,

where $\mathrm{MTTF}_{\mathrm{WS}_{4}}=\frac{1}{\lambda}=\frac{1}{0.00299}=334.45 h$ and $\mathrm{MTTR}_{\mathrm{WS} 4}=$ $3.31 \mathrm{~h}$ and $r_{\mathrm{WS} 4}=0.302$.

\section{Workstation 5}

$\mathrm{WS}_{5}$ consists of 2 machines in series as $\mathrm{WS}_{2}$; a failure of $M_{1.5}$ or $M_{2.5}$ constrains the workstation to stop. From Table 1, substituting the values of $\lambda_{i, 5}$ and $r_{i, 5}$ in Eqs. (19) and (24) we found that

$P_{0, \mathrm{wS} 5}=0.967487, \quad P_{1, \mathrm{wS} 5}=0.027773, \quad P_{2, \mathrm{wS} 5}=$ 0.004741 .

Therefore, from Eqs. (25) and (26), the availability and the unavailability of the $\mathrm{WS}_{5}$ are $A_{\mathrm{WS} 5}=96.74 \%$ and $\mathrm{UA}_{\mathrm{WS} 5}=3.26 \%$, respectively.

The reliability and the maintainability of the $\mathrm{WS}_{4}$ are: $R_{\mathrm{WS}_{5}}(t)=\exp [-0.02963 t]$ and $M_{\mathrm{WS}_{5}}(t)=1-\exp [-0.879 t]$, respectively, where $\mathrm{MTTF}_{\mathrm{WS}_{5}}=\frac{1}{\sum_{i=1}^{2} \lambda_{i, 5}}=\frac{1}{0.02963}=33.74 \mathrm{~h}$ and $\mathrm{MTTR}_{\mathrm{WS} 5}=1.13 \mathrm{~h}$ and $r_{\mathrm{WS} 5}=0.879$.

\section{Workstation 6}

The $\mathrm{WS}_{6}$ consists of 3 machines in series as $\mathrm{WS}_{1}$; a failure of $M_{1.6}$ or $M_{2.6}$ or $M_{3.6}$ constrains the workstation to stop. From Table 1, substituting the values of $\lambda_{i, 6}$ and $r_{i, 6}$ in Eqs. (19) and (24) we found that

$P_{0, \mathrm{ws} 6}=0.983537, \quad P_{1, \mathrm{ws} 6}=0.003119, \quad P_{2, \mathrm{ws} 6}=$ $0.002955, P_{3, \mathrm{ws} 6}=0.010389$.

Therefore, from Eqs. (25) and (26), the availability and the unavailability of the $\mathrm{WS}_{6}$ are $A_{\mathrm{wS} 6}=98.35 \%$ and $\mathrm{UA}_{\mathrm{Ws} 6}=1.65 \%$, respectively.

The reliability and the maintainability of the $\mathrm{WS}_{6}$ are: $R_{\mathrm{WS}_{6}}(t)=\exp [-0.0258 t]$ and $M_{\mathrm{WS}_{5}}(t)=1-\exp [-1.537 t]$, respectively, where $\mathrm{MTTF}_{\mathrm{WS}_{6}}=\frac{1}{\sum_{i=1}^{3} \lambda_{i, 6}}=\frac{1}{0.0258}=38.76 h$, then $\mathrm{MTTR}_{\mathrm{WS} 6}=0.65 \mathrm{~h}$ and $r_{\mathrm{WS} 6}=1.537$.

The reliability, availability and maintainability of the entire croissant production line, respectively, are

$$
\begin{aligned}
& R_{\mathrm{S}}(t)=\prod_{i=1}^{6} R_{\mathrm{WS}_{i}}(t)=\exp (-0.2232 t) \\
& A_{\mathrm{s}}=\prod_{i=1}^{6} A_{\mathrm{WS}_{i}}=0.84878 \\
& M_{\mathrm{S}}(t)=1-\exp (-1.3358 t) .
\end{aligned}
$$
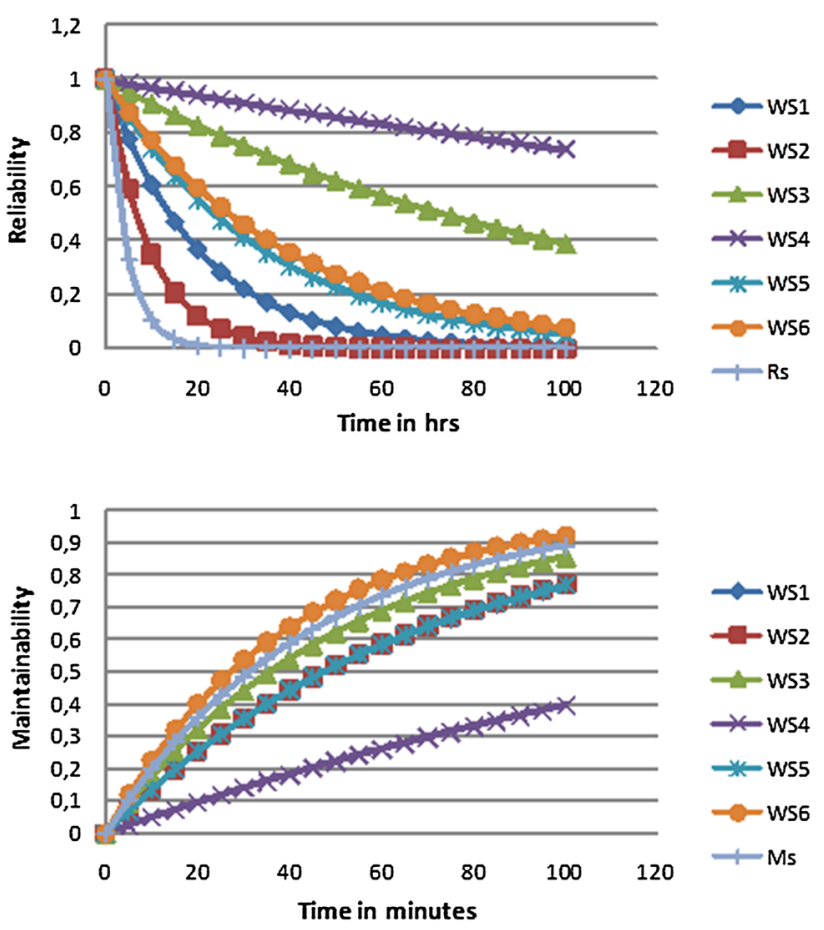

Fig. 4 Reliability and maintainability for all workstations and the entire croissant production line

In Fig. 4, the diagrams of reliability and maintainability for all workstations and the entire production system were plotted, and the following observations were made: (a) the reliability of the line in an hour of operation is $80.00 \%$, in 8 -h (a shift) of operation is $16.76 \%$ and in 24-h (a working day) of operation drops down to $0.47 \%$; (b) a failure at all workstations and for the entire line will be repaired within $100 \mathrm{~min}$, except for $\mathrm{WS}_{4}$ (baking) where the repair time is more than $500 \mathrm{~min}$. This is obvious because the maintenance staff, apart from the time it takes them to repair a failure, spends time waiting for the oven to cool down and following repair also spends time to reheat the oven; (c) the highest reliabilities are reported for $\mathrm{WS}_{4}$ (baking) and $\mathrm{WS}_{3}$ (proofing), whereas the lowest reliabilities for $\mathrm{WS}_{2}$ (forming) and $\mathrm{WS}_{1}$ (kneading); (d) the best maintainability occurs at $\mathrm{WS}_{6}$ (wrapping), whereas the worst maintainability occurs at $\mathrm{WS}_{4}$ (baking). The maintainability at $\mathrm{WS}_{1}$ (kneading), $\mathrm{WS}_{2}$ (forming) and $\mathrm{WS}_{5}$ (cooling-filling) must be revised as well; and (e) the $\mathrm{WS}_{1}$ (kneading), $\mathrm{WS}_{2}$ (forming) and $\mathrm{WS}_{5}$ (cooling-filling) $\mathrm{WS}_{6}$ (wrapping) appear to display the major unavailability of the line with $5.37,4.05,3.26$ and $1.65 \%$, respectively. 


\section{Conclusions}

Analytical probability models for an automated system that consists of $n$-machines in series with common transfer mechanism and control system are developed. The models assume both failure and repair rates to be exponentially distributed. The mathematical model of operation and the probability model of failure for the individual machine, workstation and entire system are presented. These models were applied to investigate the effect of system parameters on system performance in the actual croissant production line. It was pointed out that

(a) The availability of the line is $84.87 \%$ whereas the unavailability is $15.13 \%$. Therefore, the availability that is affected by the breakdown losses (i.e. failures) must be increased.

(b) The maintenance department may adopt the appropriate maintenance policy for the croissant production line, focusing primarily on WS1 (kneading) and $\mathrm{WS}_{2}$ (forming) that display the lowest reliability and maintainability and

(c) the reliability and maintainability diagrams at workstation and entire line level for time interval are shown.

Moreover, the steady-state probability models described in Eqs. (24), (25), and (26) can be applied in each machine consisting of individual components which fail more or less frequently, leading to failures in the equipment. Thus, it can estimate the demand for machine support/spare parts and determine the inventory management.

Open Access This article is distributed under the terms of the Creative Commons Attribution License which permits any use, distribution, and reproduction in any medium, provided the original author(s) and the source are credited.

\section{References}

Adamyan A, He D (2002) Analysis of sequential failures for assessment of reliability and safety of manufacturing systems. Reliab Eng Syst Saf 76:227-236

Born F, Criscimagna NH (1995) Translating user diagostics, reliability and maintainability needs into specification. In: Proceedings of annual reliability and maintainability symposium, IEEE, New York, NY, pp 106-111

Brischke IWR, Murthy DNP (2003) Case study in reliability and maintenance. Wiley, Hoboken, New Jersey, USA

Burman M, Gershwin SB, Suyematsu C (1998) Hewlett-Packard uses operations research to improve the design of a printer production line. Interfaces 28(1):24-26

Buzacott JA (1968) Prediction of the efficiency of production systems without internal storage. Int J Prod Res 6:173-188

Buzacott JA, Shanthikumar JG (1993) Stochastic models of manufacturing systems. Prentice-Hall, Englewood Cliffs

Chen CT, Yuan J (2004) Transient throughput analysis for a series type system of machines in terms of alternating renewal processes. Eur J Oper Res 155:178-197
Chiang SY, Kuo SY, Meerkov SM (1998) Bottlenecks in Markovian production line: a systems approach. IEEE Trans Robot Autom 14(2):352-359

Cockerill AW (1990) RAM analysis helps cut turbine-generator systems costs. Power Eng 94(7):27-29

Collas B (1994) Reliability and availability estimation for complex system: a simple concept and tool. In: Proceedings of annual reliability and maintainability symposium, IEEE, New York, NY, pp 110-113

Dallery Y, Gershwin SB (1992) Manufacturing flow line systems: a review of models and analytical results. Queueing Syst 12(1-2):3-94

Dhillon BS (2006) Maintainability, maintenance and reliability for engineers. Taylor \& Francis Group, Boca Raton, FL, USA

Dhouib K, Gharbi A, Ayed S (2008) Availability and throughput of unreliable, unbuffered production lines with non-homogeneous deterministic processing times. Int J Prod Res 46(20):5651-5677

Dogan E, Altiok T (1998) Blocking policies in pharmaceutical transfer lines. Ann Oper Res 79:323-347

Ebeling CE (1997) An Introduction to reliability and maintainability engineering. McGraw Hill, New York

Faghihinia E, Mollaverdi N (2012) Building a maintenance policy through a multi-criterion decision-making model. J Ind Eng Int 8:14

Freiheit T, Shpitalni M, Hu SJ, Koren Y (2004) Productivity of synchronized serial production lines with flexible reserve capacity. Int J Prod Res 42(10):2009-2027

Gershwin SB (1994) Manufacturing systems engineering. PrenticeHall, Englewood Cliffs

Glassey C, Hong Y (1993) The analysis of behavior of an unreliable $n$-stage automatic transfer line with $(n-1)$ interger-stage storage buffers. Int J Prod Res 31(3):519-530

Golrizgashti S (2014) Supply chain value creation methodology under BSC approach. J Ind Eng Int 10:67

Govil MC, Fu MC (1999) Queuing theory in manufacturing: a survey. J Manuf Syst 18(3):214-240

Hassett TF, Dietrich DL, Szidarovszky F (1995) Time-varying failure rates in the availability and reliability analysis of repairable systems. IEEE Trans Reliab 44(1):155-160

Hou W, Okogbaa OG (2002) A simplified availability modeling tool for networks with 1:1 redundant software-hardware systems. In: Proceedings of reliability and maintainability symposium, IEEE, New York, NY, pp 556-562

Jin T, Janamanchi B, Feng Q (2011) Reliability deployment in distributed manufacturing chains via closed-loop Six Sigma methodology. Int J Prod Econ 130(1):96-103

Karim MA, Smith AJR, Halgamuge S (2008) Empirical relationships between some manufacturing practices and performance. Int $\mathrm{J}$ Prod Res 46(13):3583-3613

Kim J, Geshwin SB (2005) Integrated quality and quantity modelling of a production line. OR Spectr 27(2-3):287-314

Koulamas CP (1992) A stochastic model for a machining cell with tool failure and tool replacement considerations. Comput Oper Res 19:717-729

Lai CD, Xie M, Poh KL, Dai YS, Yang P (2002) A model for availability analysis of distributed software/hardware system. Inf Softw Technol 44:343-350

Liberopoulos G, Kozanidis G, Tsarouhas P (2007) Performance evaluation of an automatic transfer line with WIP scrapping during long failures. M\&SOM 9(1):62-83

Lin Y (2009) System reliability evaluation for a multistate supply chain network with failure nodes using minimal paths. IEEE Trans Reliab 58:34-40

Markeset T, Kumar U (2001) R\&M and risk-analysis tools in product design, to reduce life cycle cost and improve product attractiveness. In: Proceedings of annual reliability and maintainability symposium, IEEE, New York, NY, pp 116-121 
Marseguerra M, Zio E, Podofillini L (2004) Optimal reliability/ availability of uncertain systems via multi-objective genetic algorithms. IEEE Trans Reliab 53(3):424-434

Papadopoulos HT, Heavey C (1996) Queueing theory in manufacturing systems analysis and design: a classification of models for production and transfer lines. Eur J Oper Res 92:1-27

Patti LA, Watson JK (2010) Downtime variability: the impact of duration-frequency on the performance of serial production systems. Int J Prod Res 48(19):5831-5841

Pecht M (2009) Product reliability, maintainability, and supportability handbook, 2nd edn. Taylor \& Francis Group, LLC, Boca Raton, FL, USA

Raissi S, Gatmiry Z (2012) A Six Sigma approach to boost up time domain reliability in multi-stage services. Afr J Bus Manag 6(4):1367-1374

Rezg N, Xie X, Mati Y (2004) Joint optimization of preventive maintenance and inventory control in a production line using simulation. Int J Prod Res 42(10):2029-2046

Savsar M (2000) Reliability analysis of a flexible manufacturing cell. Reliab Eng Syst Saf 67(1):147-152

Sharma RK, Kumar D, Kumar P (2008) Fuzzy modelling of system behaviour for risk and reliability analysis. Int J Syst Sci 39(6):563-581

Simeu-Abazi Z, Daniel O, Descotes-Genon B (1997) Analytical method to evaluate the dependability of manufacturing systems. Reliab Eng Syst Saf 55:125-130

Srinivasa Rao M, Naikan VNA (2014) Reliability analysis of repairable systems using system dynamics modeling and simulation. J Ind Eng Int 10:69

Taghizadeh H, Hafezi E (2012) The investigation of supply chain's reliability measure: a case study. J Ind Eng Int 8:22

Tan B (1999) Variance of the output as a function of time: production line dynamics. Eur J Oper Res 117(3):470-484
Tatry PH, Deneu F, Siomonotti JL (1997) RAMS approach for reusable launch vehicle advanced studies. Acta Astronaut 41(11):791-797

Tsarouhas P (2012) Reliability, availability, and maintainability (RAM) analysis in food production lines: a review. Int J Food Sci Technol 47(11):2243-2251

Tsarouhas P (2013) Reliability analysis into hospital dialysis system: a case study. Qual Reliab Eng Int 29(8):1235-1243

Xie XL (1993) Performance analysis of a transfer line with unreliable machines and finite buffers. IIE Trans 25:99-108

Yusuf I (2014) Comparative analysis of profit between three dissimilar repairable redundant systems using supporting external device for operation. J Ind Eng Int 10:77

Dr. Panagiotis H. Tsarouhas Dipl. Eng., Ph.D. is Assistant Professor at Technological Educational Institute of Central Macedonia (Greece), Department of Supply Chain Management and Logistics and a tutor in postgraduate course on Quality Assurance in Hellenic Open University. He received his Diploma in Mechanical Engineering from Universita degli studi di Napoli Federico II (Italy) and both Ph.D. and M.Sc. from the Department of Mechanical and Industrial Engineering from the University of Thessaly in Volos, Greece. For about 10 years he was at the Technical Department as production and maintenance operation head in food industry 'Chipita International $\mathrm{SA}^{\prime}$ ' in Lamia, Greece. He has about 23 years of research/teaching experience and more than 40 research papers in international journals, book chapters and conference proceedings. He has been involved in many research and practical projects in the field of reliability analysis and maintenance engineering. His areas of interest are reliability and maintenance engineering, quality engineering and supply chain management. 